\title{
Getting the most out of historic reliability data
}

DOI:

10.1109/PHM.2012.6228953

Link to publication record in Manchester Research Explorer

\section{Citation for published version (APA):}

Feng, D., Wang, Z., \& Jarman, P. (2012). Getting the most out of historic reliability data. In Prognostics and System Health Management (PHM), 2012 IEEE Conference on (pp. 1-6) https://doi.org/10.1109/PHM.2012.6228953

\section{Published in:}

Prognostics and System Health Management (PHM), 2012 IEEE Conference on

\section{Citing this paper}

Please note that where the full-text provided on Manchester Research Explorer is the Author Accepted Manuscript or Proof version this may differ from the final Published version. If citing, it is advised that you check and use the publisher's definitive version.

\section{General rights}

Copyright and moral rights for the publications made accessible in the Research Explorer are retained by the authors and/or other copyright owners and it is a condition of accessing publications that users recognise and abide by the legal requirements associated with these rights.

\section{Takedown policy}

If you believe that this document breaches copyright please refer to the University of Manchester's Takedown Procedures [http://man.ac.uk/04Y6Bo] or contact uml.scholarlycommunications@manchester.ac.uk providing relevant details, so we can investigate your claim.

\section{OPEN ACCESS}




\title{
Getting the Most Out of Historic Reliability Data
}

\author{
Stastistical Analysis on the UK Transmission Power Transformer Population
}

\author{
Dongyin Feng and Zhongdong Wang* \\ The University of Manchester \\ Manchester M60 1QD, UK \\ "zhongdong.wang@manchester.ac.uk
}

\author{
Paul Jarman \\ The National Grid Company \\ Warwick CV34 6DA, UK
}

\begin{abstract}
In this paper, an intensive statistical analysis on the UK transmission power transformer historic reliability data is presented. It is shown that deriving the reliability of transformer at a certain age by simply calculating the hazard rate is inadequate, as the hazard rate in each age has a statistical range in which the confidence band width is related to the amount of the reliability data involved in that age. Since the post-mortem investigations on the failed units have verified that all failures up till now follow random failure mechanism, a general hazard rate of $0.27 \%$ with standard deviation of $0.03 \%$ has been derived for the UK transmission power transformers based on all the reliability data available. In term of data sufficiency, as supported by the hazard rate's confidence band analysis, only if the number of transformer at one age is more than 200 should the calculated hazard rate be reliable.
\end{abstract}

Keywords-transformer reliability; hazard rate; poisson distribution; confidence band

\section{INTRODUCTION}

As the most essential apparatus in the power network, the failure-in-service of a power transformer could lead to the power supply interruption and the capital replacement cost. Although the expected lifetime of a power transformer has been recognized as approximately 40 years, the majority of the transmission power transformers at the UK power network were installed during the 1960 s, and the historic reliability data so far does not show any increase in the failure trend among the transformer aged 40 years or plus, making this life approximation in doubt [1].

As a consequence an alternative route has been taken to understand the reliability of the transformer population through examining the health status of the individual units. Recognizing that the life expectation has been exceeded by many aged power transformers in service, utilities have gradually diverted their interests into diagnosing and monitoring the condition of the critical units. A scoring system has been developed to take into considerations of variety aspects of individual unit such as DGA results, oil quality, component conditions, load history, design problem and so on to determine the unit's reliability in the near future [2-4].

However for long term planning, as the population ages, a replacement peak would be foreseen projected by the peak installation period during the 1960s, which requires a much more advanced schedule for the capital re-investment. In dealing with such issue, it is of asset managers' desire to have an accurate transformer life model. Attempts have been made based on the available historic reliability data to gain the information on the population's failure or replacement rate for the sake of developing the replacement strategy [5-7]. To this end, different mathematic models [8-11] have been proposed to fit the transformer reliability data to predict the future failure rate. However the models' practical uses are in debate as their outputs are very much affected by the limited data in older transformer ages.

This paper presents the statistical analysis performed on the historic reliability data of the UK transmission power transformer population. To give a general view of the transformer hazard rate per age, the hazard plot of each transformer age is presented which is derived based on the demographic data of in-service and failure unit number. Under each age, the hazard rate's 95\% confidence band is quantified via the use of Binomial and Poisson distribution to calculate the likelihoods of different hazard rates. By the support of the postmortem investigations on the failed unites which have verified that all failures up till now follow the random failure mechanism, a general hazard rate and its standard deviation is derived by utilizing all the reliability data available. The data sufficiency for a transformer age is also discussed in this paper.

\section{TRANSFORMER DEMOGRAPHIC AND HAZARD PlOT}

The United Kingdom is one of the few pioneering countries to lead the electrification. The installation of transmission power transformers dates back to as early as 1952 . Looking at the reliability data in 2010, the oldest unit in service is 57 years old. The transformer demographic data are plotted according to the transformer age as in Fig. 1 and 2 to give a general view on the in-service (i.e. survivor) and failure number at each individual age.

According to [1, 12], the definitions of the survivor and failure number at transformer Age $t$ are stated respectively as the number of transformers that have survived $t$ years, and the number of transformers that have failed during Age $t$. The rules of computing survivor and failure number are:

- As long as a transformer was installed and commissioned to the network and has survived the first year of its service, it contributes to the bin of survivor number in Age 0. For any additional years of its 
surviving, the contribution would be counted in the further corresponding age bins.

- If a transformer fails after $t$ years since its installation, this failure is marked in the bin of failure number at Age $t$. Meanwhile this particular transformer would also be spotted in the survivor bins from Age 0 till Age $t-1$. For those transformers which have been well operating since installation, they contribute into the survivor bins from Age 0 all the way till their age bins calculated.

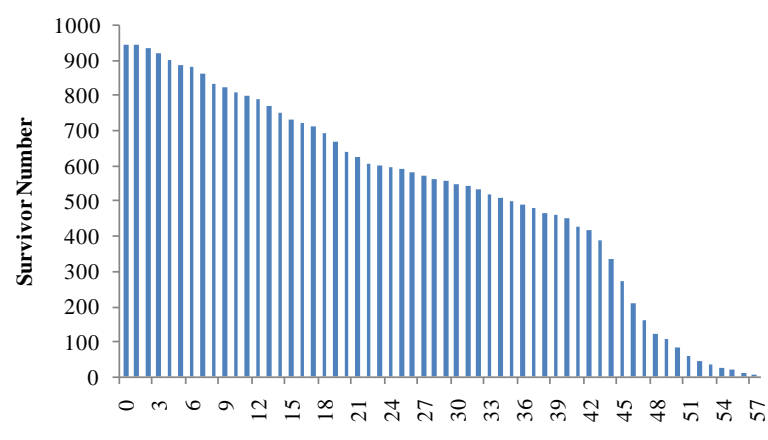

Transformer Age

Figure 1. The UK transmission power transformer's survivor number from Age 0 to 57 .

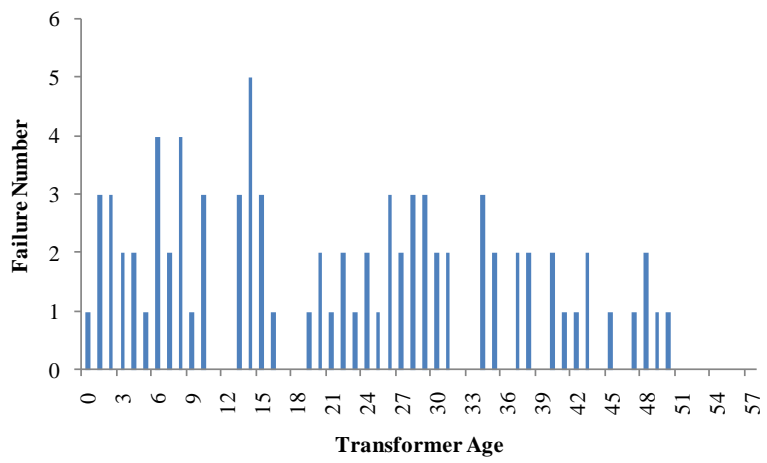

Figure 2. The UK transmission power transformer's failure number from Age 0 to 57 .

Fig. 1 illustrates the obvious decrease trend on the survivor number as transformer age increases. More than 900 power transformers have survived their first year of operation, while on the other end seven transformers with the record age of 57 years are still operating in the network. The survivor number starts to decrease drastically from Age 47.

In Fig. 2, the failure number of the UK transmission power transformer population appears to be randomly distributed within 5. In fact, as the UK National Grid has been proactively retiring transformers with poor conditions, this action effectively truncates the failure number in old ages and results in lower-than-expected trend of figures.

It is worth pointing out that throughout nearly 60 years of operation, the UK National Grid has performed thorough postmortem investigations on the failed units $[13,14]$. The results have shown that up till now, all units have failed due to the random failure mechanism except one particular unit which failed due to the age-related insulation deterioration. Considering the coherence of the failure mechanism in the reliability data, the age-related failure unit has been removed and is not included in Fig. 2.

The reliability of transformer over age is usually presented as the transformer hazard rate per age. In reliability analysis, the term hazard rate is frequently used to measure the sample's proneness to failure as a function of age [15]. At each age, transformer's hazard rate $h(t)$ can be calculated using (1):

$$
h(t)=\frac{\text { failure }(t)}{\text { survivor }(t)}
$$

where failure(t) and survivor(t) denote the number of failure and survivor at Age $t$ respectively, and $h(t)$ is the corresponding hazard rate. Using (1) the hazard plot of all transformer ages is shown in Fig. 3.

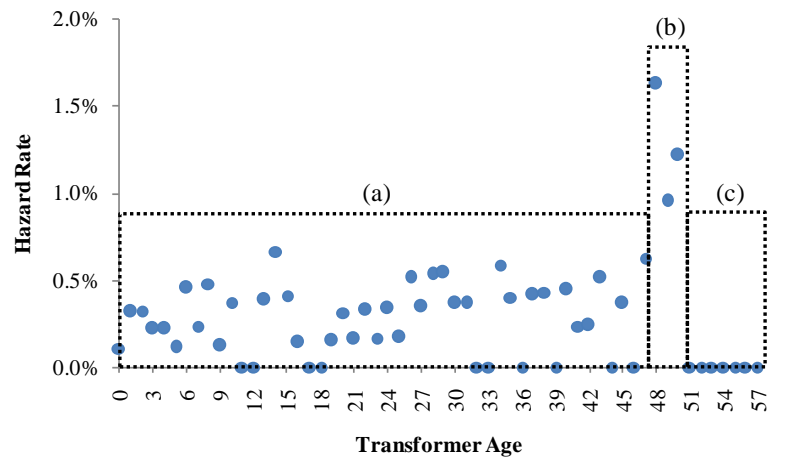

Figure 3. The UK transmission power transformer's hazard rate from Age 0 to 57

The hazard rates shown in Fig. 3 are observed to be never exceeding $2 \%$. The entire age span is split into three regions as:

- Region (a) consists of ages from 0 to 46 , in which the hazards are fluctuating within the level of $0.8 \%$. In these ages the transformer survivor numbers are fairly big (i.e. larger than 200). The fluctuation is caused by the different failure number, i.e. the numerator in (1).

- Region (b) consists of ages from 47 to 50, where the hazards increase up to $1.6 \%$. The reason to this sudden increase is the drastic decrease of the survivor numbers. This effectively results in small denominator in (1), and hence very large hazard rates.

- $\quad$ Region (c) consists of the old ages from 51 to 57. In this range the hazards are $0 \%$, because no failure has been observed among the very small transformer survivor population in these ages.

It is seen from (1) that the hazard rate is affected by both failure and survivor number. Looking at both Age 10 and 45 as examples: the hazard rates at these two ages are the same $(0.37 \%)$, but the combinations of failure and survivor numbers are different (i.e. 3 failures over 811 survivors at Age 10; and 1 failure over 273 survivors at Age 45). Under this circumstance 
although the hazard rates are derived to be the same, each age involves different amount of failure and survivor data. Obviously earlier transformer ages involve more data while on the other hand data shortage is seen at the older ages.

From statistical point of view, only if the data is sufficient should the analysis result (e.g. the hazard rate per age) be considered as reliable and hence is acceptable [16]. In this sense different transformer age's hazard rate must yield different level of confidence. As further analysis, the likelihood of the hazard rate at each individual transformer age is computed in the next section.

\section{HAZARD RATE'S 95\% CONFIDENCE BAND}

In this section, the $95 \%$ confidence band of each individual age's hazard rate is quantified via assessing hazard rate's likelihood by Binomial and Poisson distribution.

The statistical term of 'likelihood' should be distinguished from the more frequently used term of 'probability' [15, 17]. The latter one refers to the measure of how certain, or uncertain, an outcome occurs out of an event. It can be understood as a parameter that exists or can be derived before the occurrence of the event. The term 'likelihood' opposes 'probability' in such way that it is a measure of the event's parameter after the occurrence of the event. As a simple example, in the event of coin tossing, $P(H H \mid p=0.5)$ denotes the 'probability' of observing two heads consequently giving that the chance of having head and tail from this coin is 50-50; while $L(p=0.5 \mid H H)$ denotes the 'likelihood' of head-to-tail ratio being $50 \%$ giving that the two heads have been consequently observed. In this sense the term 'likelihood' can be understood as the probability of 'probability'. In this paper, it is the hazard rate's 'likelihood' that will be examined.

The evaluation of the likelihood starts with the probability calculation. In the reliability analysis at any transformer age, the probability of failure occurrence can be calculated using either Binomial or Poisson distribution as in (2) and (3).

$$
\begin{gathered}
P(f \mid n, h)=\frac{n !}{f !(n-f) !} h^{f}(1-h)^{n-f} \\
P(f \mid \lambda)=\frac{\lambda^{f} \exp ^{-\lambda}}{f !}
\end{gathered}
$$

In Binomial distribution i.e. (2), $P(f \mid n, h)$ is the conditional probability of having $f$ numbers of failure, given that the survivor number is $n$ and the expected hazard rate is $h$. In Poisson distribution i.e. (3), $P(f \mid \lambda)$ is the conditional probability of having $f$ numbers of failure, given that the expected failure number is $\lambda$ which is the product of $h$ and $n$ as in Binomial distribution. When $h$ is small, both Binomial and Poisson distributions provide almost exactly the same results $[15,16]$.

To examine hazard rate's likelihood $L\left(\lambda^{\prime} \mid f\right)$, the use of Bayes Theorem is an effective approach [18], which has the mathematical expression shown in (4) and (5) depending on which distribution is used. Note the superscripts in $f^{\prime}$ and $\lambda$ ' denote the hazard rate which is artificially altered in a range in order to examine the corresponding likelihood:

$$
\begin{aligned}
L\left(h^{\prime} \mid f\right) & =\frac{P\left(f \mid h^{\prime}\right) P\left(h^{\prime}\right)}{P(f)} \\
L\left(\lambda^{\prime} \mid f\right) & =\frac{P\left(f \mid \lambda^{\prime}\right) P\left(\lambda^{\prime}\right)}{P(f)}
\end{aligned}
$$

In (4) and (5), $P\left(h^{\prime}\right), P\left(\lambda^{\prime}\right)$ and $P(f)$ are the prior probabilities of $h^{\prime}, \lambda^{\prime}$ and $f$. Without any prior knowledge of transformer failure statistics, $P\left(h^{\prime}\right), P\left(\lambda^{\prime}\right)$ and $P(f)$ are uniformly distributed from $0 \%$ to $100 \%$, and are therefore canceled and eliminated in the equations. The procedures of evaluating the likelihoods $L\left(h^{\prime} \mid f\right)$ and $L\left(\lambda^{\prime} \mid f\right)$ are effectively the calculation of the probabilities $P\left(f \mid h^{\prime}\right)$ and $P\left(f \mid \lambda^{\prime}\right)$ using Binomial and Poisson distribution respectively.

In each age, the hazard rate is increased from $0 \%$ to $100 \%$ in steps of $0.05 \%$ to calculate the likelihood density of $L(h ' f)$ or $L\left(\lambda^{\prime} \mid f\right)$, followed by the plot of the cumulative likelihood density. The hazard rate's $95 \%$ confidence band is thereby extracted from the cumulative density in between the cumulative density of $2.5 \%$ and $97.5 \%$, which correspond to the lower and upper limit of the hazard rate.

As an example, the likelihood density $L\left(\lambda^{\prime} \mid f\right)$ calculated by Poisson distribution and the cumulative density plot in Age 0 are shown in Fig. 4 and 5 below.

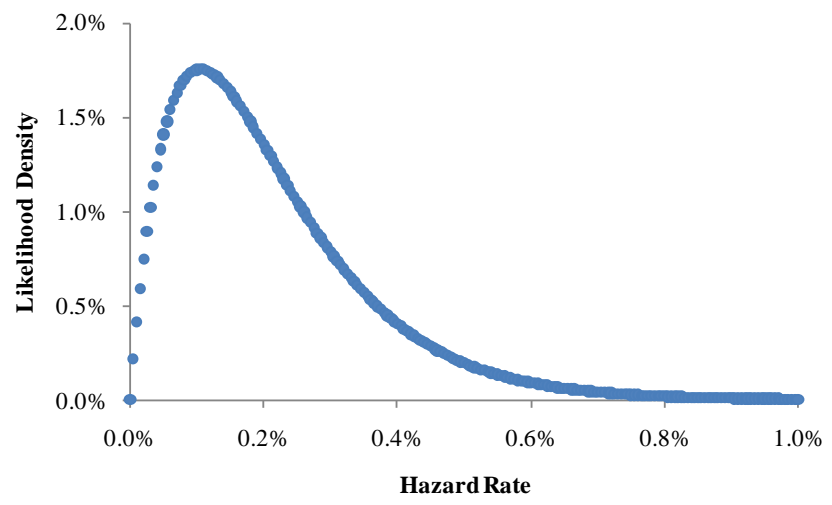

Figure 4. Hazard rates' likelihood density in Age 0 (shown from 0 to $1.0 \%$ ).

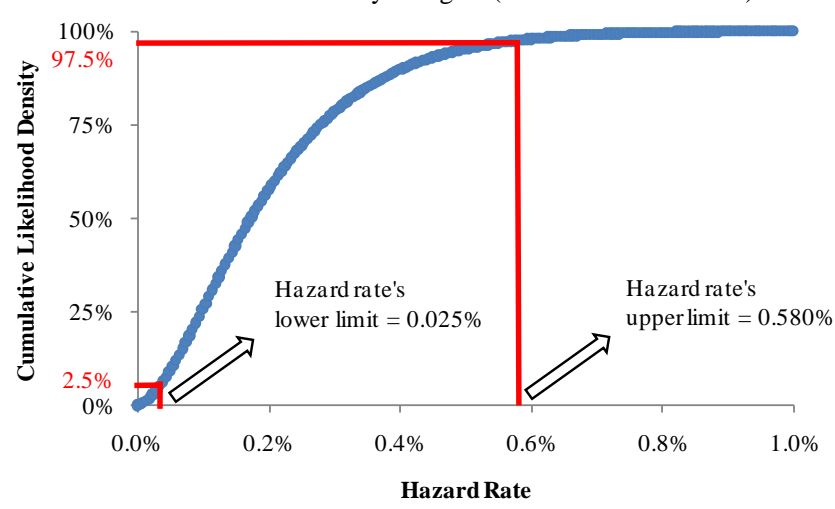

Figure 5. Hazard rates' likelihood cumulative density in Age 0, and the extraction of the $95 \%$ confidence band (shown from 0 to $1.0 \%$ ). 
As can be observed in Fig. 4, in the range of 0 to $100 \%$, the hazard rate bearing the highest likelihood is $0.105 \%$ which is the true hazard rate in Age 0 (1 failure and 949 survivors). However the likelihood density plot reveals the fact that from likeliness point of view, the likelihood of the hazard rate $0.105 \%$ does not dominate over the other hazard rates nearby. By extracting the hazard rate's 95\% confidence band on Fig. 5, the cumulative likelihood density plot, it is learnt that the hazard rate in Age 0 is statistically spread from $0.025 \%$ to $0.58 \%$.

Fig. 4 and 5 provide straightforward illustrations on how hazard rates' likelihoods distribute, and the procedure on the confidence band extraction. However the precision on the extracted confidence band is limited by the resolution in the likelihood calculation, i.e. the $0.005 \%$ increment in the hazard rate. Furthermore, the procedure is rather complicated such that the likelihoods must be calculated over a broad range. As improvement, the following expressions (6)-(9) are introduced to directly calculate the failure numbers corresponding to the likelihood cumulative density of $2.5 \%$ and $97.5 \%$, and the conversion to the hazard rate. In fact, only calculating the confidence band using Poisson distribution could have such simplified expression, due to the simplicity of the formula Poisson distribution has.

$$
\begin{gathered}
\frac{\int_{0}^{x_{\text {lover }}} \frac{\lambda^{f} \exp ^{-f} d \lambda}{f !}}{\int_{0}^{N} \frac{\lambda^{f} \exp ^{-f} d \lambda}{f !}}=\frac{\int_{0}^{x_{\text {lower }}} \lambda^{f} \exp ^{-f} d \lambda}{\int_{0}^{N} \lambda^{f} \exp ^{-f} d \lambda}=2.5 \% \\
\frac{\int_{0}^{x_{\text {uper }}} \frac{\lambda^{f} \exp ^{-f} d \lambda}{f !}}{\int_{0}^{N} \frac{\lambda^{f} \exp ^{-f} d \lambda}{f !}}=\frac{\int_{0}^{x_{\text {upper }}} \lambda^{f} \exp ^{-f} d \lambda}{\int_{0}^{N} \lambda^{f} \exp ^{-f} d \lambda}=97.5 \% \\
h_{\text {lower }}=\frac{x_{\text {lower }}}{N} \\
h_{\text {upper }}=\frac{x_{\text {upper }}}{N}
\end{gathered}
$$

In (6) and (7), $x$ and $N$ are the failure and surviving number. The numerators in (6) and (7) calculate the cumulative likelihood density of the failure number from 0 to $x$, while the denominators are effectively accumulating the likelihoods of failures from 0 to $N$. Equating the formulae to $2.5 \%$ and $97.5 \%$, the lower and upper limit value of $x$ can be calculated. The corresponding hazard rate can be converted from $x$ using (8) and (9).

The confidence band of the hazard rate over the transformer age is shown in Fig. 6. Note that the discrete likelihood points have been drawn as solid lines to illustrate the range.

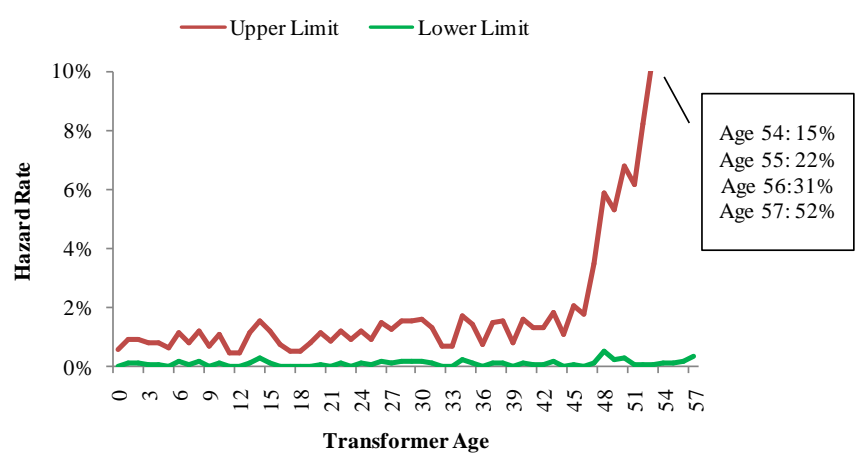

Figure 6. Hazard rate's $95 \%$ confidence bands in all transformer ages.

According to Fig. 6, the hazard's 95\% confidence band can be roughly split into two regions, in which the boundary is Age 46. In the first half of the figure, the upper limits of hazard rate retain within $2 \%$.

Beyond Age 46, the band's upper limits start to increase into whopping high levels. In an extreme case, the hazard's upper limit at Age 57 indicates that even a hazard rate as high as $52 \%$ is statistically possible. However such appearance in hazard's confidence band does not suggest any increasing in transformer failure risk as age progresses, but is purely due to the lack of data in older ages.

The analysis on hazard rate's $95 \%$ confidence band has reveal the fact that the confidence, or the uncertainty in the hazard rate determination is statistically affected by the sample size the age subjects to. The bigger the sample size, the smaller the possible range the hazard rate has.

\section{DISCUSSION}

In previous sections, the hazard rate has been proved to be inadequate in representing the transformer age's reliability. The hazard rate's $95 \%$ confidence band has been quantified and has shown that the possible range in hazard rate is related to the size of the sample data. Based on these findings, this section aims to discover meaningful statistical results from the UK transmission power transformer historic reliability data, to serve the ultimate goal of assisting the development of the asset management strategy.

\section{A. Confidence Band Analysis on Different Age Intervals}

To study the impact the increasing sample size has on the hazard rate's likelihood, the transformer reliability data are grouped at every 3- and 5-year interval, i.e. the survivor and failure numbers are summed respectively in Age 0 to 2, 3 to 5 and 6 to 8 onwards in the case of 3 -year interval, and Age 0 to 4,5 to 9 and 10 to 14 onwards in the case of 5 -year interval. The hazard rate in each interval is derived accordingly using the summed failure number divided by the summed survivor number. The procedure in calculating hazard rate's likelihood has been introduced in section III.

As examples, three groups in each interval study have been selected and presented in this paper. The reliability data of each 
group are listed in TABLE I, and the hazard rate's likelihood plot is shown in Fig. 7.

TABLE I. RELIABILITY DATA OF SIX GROUPS

\begin{tabular}{|c|c|c|c|c|}
\hline \multirow{2}{*}{ Group } & \multirow{2}{*}{$\begin{array}{c}\text { Age } \\
\text { Involved }\end{array}$} & \multicolumn{4}{|c|}{ Three-year Interval } \\
\cline { 3 - 5 } & $\begin{array}{c}\text { Survivor } \\
\text { Number }\end{array}$ & $\begin{array}{c}\text { Failure } \\
\text { Number }\end{array}$ & $\begin{array}{c}\text { True Hazard } \\
\text { Rate }\end{array}$ \\
\hline $\mathbf{1}$ & $\mathbf{1 8 - 2 0}$ & 2006 & 3 & $0.15 \%$ \\
\hline $\mathbf{2}$ & $\mathbf{3 6 - 3 8}$ & 1437 & 4 & $0.28 \%$ \\
\hline $\mathbf{3}$ & $\mathbf{6 - 8}$ & 2583 & 10 & $0.39 \%$ \\
\hline & & & \multicolumn{3}{|c|}{ Five-year Interval } \\
\hline $\mathbf{4}$ & $\mathbf{1 5 - 1 9}$ & 3536 & 5 & $0.14 \%$ \\
\hline $\mathbf{5}$ & $\mathbf{5 - 9}$ & 4296 & 12 & $0.28 \%$ \\
\hline $\mathbf{6}$ & $\mathbf{2 5 - 2 9}$ & 2868 & 12 & $0.42 \%$ \\
\hline
\end{tabular}

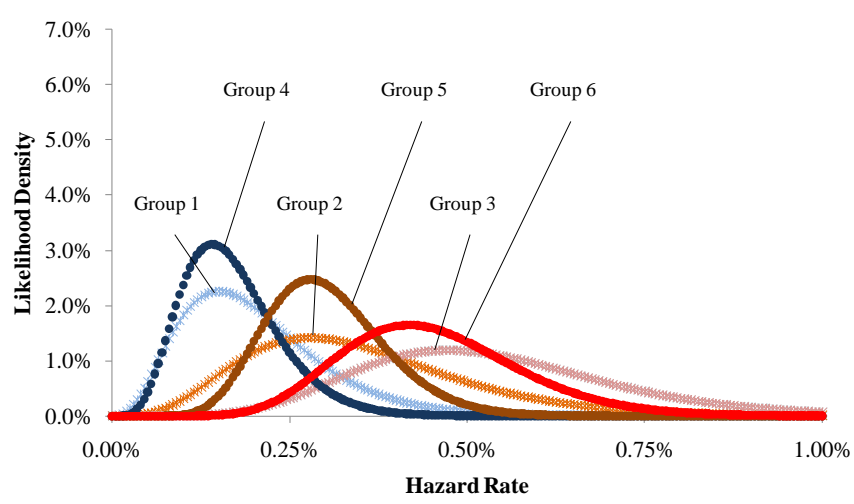

Figure 7. Likelihood density on the hazard rates after grouping 3 (group 1-3) and 5 (group 4-6) transformer ages' reliability data.

Comparing the likelihood density of the hazard rates among Group 1 and 4, it can be seen that although the hazard rates are almost the same, as Group 4 has involved more data, the possible range of the hazard rate is more restricted than Group 1. The likelihood of the peak hazard rate is more outstanding in Group 4 than 1. Same observations can be made if comparing Group 5 and 6 with 2 and 3.

Since this study has confirmed that the more reliability data involved the more restricted the confidence band of the hazard rate is, to eliminate the negative impact of the limited data and hence towards the most confident result, the reliability data in all transformer ages are grouped together to derive a general hazard rate. In fact this is a feasible approach considering the same failure mechanism (i.e. random, non age-related) of all the failed units as verified by the post-mortem investigations.

The general hazard rate of the UK transmission power transformer population is calculated as equals to $0.27 \%$. As comparison, the likelihood of the general hazard rate is plotted with all the cumulative likelihoods in Fig. 7 to illustrate the greatest confidence of the general hazard rate, as shown in Fig. 8.

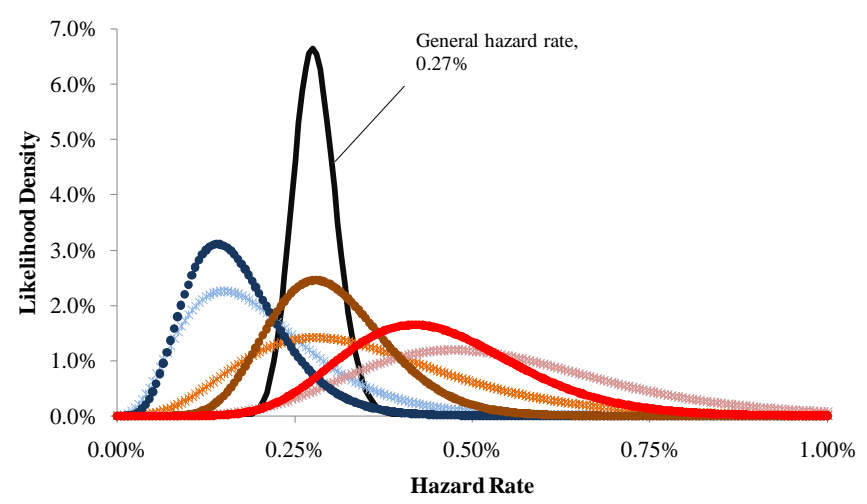

Figure 8 . Likelihood density on general hazard rate of $0.27 \%$ comparing with the 6 groups' likelihood plot.

Obviously by grouping the data together, the general hazard rate has the most restricted range than any of the subgroups, and it has the lower and upper limits of $0.21 \%$ and $0.33 \%$ for the confidence band of $95 \%$ respectively. Furthermore, as the pattern of the general hazard rate appears to be normal distribution, the approximation indicates that the standard deviation equals to $0.03 \%$.

\section{B. Data Sufficiency In Statistical Analysis}

With the concern of the limited reliability data in older transformers, the question of 'how much data is considered sufficient for a reliable statistical analysis' naturally arises itself. The answer of such question very much relies on statistician's subjective judgments.

As in the case of the statistical analysis on the UK transmission power transformer reliability data, the question regarding the data sufficiency shall be answered through the hazard rate's 95\% confidence band as shown in Fig. 6. Only if the transformer age involves the survivor unit number of at least 200, should the statistical analysis results (in this case the hazard rate per age) be reliable and acceptable. This statement is established based on the observation that the hazard rate's upper limit has never exceeded 2\% until Age 46 (included), in which age the survivor number is 209. Beyond Age 46, the survivor number drops below 200 and more importantly the upper limit of the confidence band starts to drastically increase.

\section{CONCLUSION}

In this paper, an intensive statistical analysis has been performed on the UK transmission power transformer historic reliability data, aiming to extract valuable information.

Although the hazard rate per transformer age is fairly easy to calculate, the hazard rate's $95 \%$ confidence band has indicated that it can increase into unreliable levels as the sample size reduces in older transformer ages.

Grouping the transformer data in different ages has verified that the more data involved, the more confident the hazard rate would be. As the consequence of all failures ovserved so far are non age-related, a general hazard rate of $0.27 \%$ having 
standard deviation of $0.03 \%$ is derived by grouping all ages' reliability data together.

From the data sufficiency point of view, as indicated by the hazard rate's $95 \%$ confidence band, only if one transformer age having survivor number of at least 200 should the calculated hazard rate be regarded as reliable and hence is acceptable.

\section{ACKNOWLEDGMENT}

The authors would like to express their sincere gratitude to the UK National Grid Company for the technical and financial supports to the research on "Transformer end-of-life modeling" at the University of Manchester. Dongyin Feng would like to express his gratitude to the UK National Grid Company for partially funding for his $\mathrm{PhD}$ research study.

\section{REFERENCES}

[1] D. Y. Feng, W. Wu, Z.D. Wang and P. Jarman, "Transmission transformer end-of-life modelling: incorporating insulating paper's thermal lifetime analysis with ordinary statistical analysis," in 17th International Symposium on High Voltage Engineering, Hannover, Germany, 2011.

[2] A. Jahromi, R. Piercy, S. Cress, J. Service and W. Fan, "An approach to power transformer asset management using health index," Electrical Insulation Magazine, IEEE, vol. 25, pp. 20-34, 2009.

[3] J. Lapworth and A. Wilson, "The asset health review for managing reliability risks associated with ongoing use of ageing system power transformers," in International Conference on Condition Monitoring and Diagnosis, Beijing, 2008, pp. 605-608.

[4] D. Hughes, "The use of health indices to determine end of life and estimate remnant life for distributionassets," in 17th International Conference on Electricity Distribution, Barcelona, 2003, pp. 1-5.15.

[5] C. Kurtz, G. Ford, M. Vainberg, M. Lebow and B. Ward, "Managing Aged Transformers-Utility develops repair/refurbish/replace strategy using innovative risk-based methodologies," Transmission and Distribution World, vol. 57, pp. 30-43, 2005.

[6] N. A. J. Hastings, Physical asset management. Springer Verlag London, 2009.

[7] T. Nakagawa, Advanced reliability models and maintenance policies. Springer Verlag, 2008.

[8] J. Cota-Felix, F. Rivas-Davalos and S. Maximov, "A new method to evaluate mean life of power system equipment," in CIRED 20th International Conference and Exhibition, Prague, Czech Republic, 2009.

[9] R. Jongen, P. Morshuis, J. Smit, A. Janssen and E. Gulski, "A statistical approach to processing power transformer failure data," in 19th International Conference on Electricity Distribution, Vienna, 2007.

[10] W. Li, "Evaluating mean life of power system equipment with limited end-of-life failure data," IEEE Transactions on Power Systems, vol. 19, pp. 236-242, 2004.

[11] W. Bartley, "Analysis of transformer failures," in International Association of. Engineering Insurers, 36th Annual Conference, Stockholm, 2003.

[12] Q. Zhong, Z.D. Wang and P. Crossley, "Power transformer end-of-life modelling: linking statistical approach with physical ageing process," in CIRED Workshop, Lyon, France, 2010.

[13] P. Jarman, R. Hooton, L. Walker, Q. Zhong, T. Ishak and Z.D. Wang, "Transformer life prediction using data from units removed from service and thermal modelling," in CIGRE Sesson 2010, Paris, France, 2010.

[14] P. Jarman, Z.D. Wang, Q. Zhong and T. Ishak, "End-of-life modelling for power transformers in aged power system networks," in CIGRE 2009 6th Southern Africa Regional Conference, Cape Town, Southern Africa, 2009.

[15] W. Nelson, Applied life data analysis. John Wiley \& Sons Inc, 1982.

[16] S. L. Meyer, Data analysis for scientists and engineers. New York: Wiley, 1975, vol. 1, 1975.

[17] L. B. Page, Probability for engineering with applications to reliability. Computer Science Press, 1989.

[18] J. Cornfield, "Bayes theorem," Revue de l'Institut International de Statistique, pp. 34-49, 1967. 